\title{
An unusual gait disorder at the Emergency Department: role of the quantitative assessment of parenchymal transcranial Doppler sonography
}

\author{
Massimiliano Godani ${ }^{1 \#}$, Giuseppe Lanza $^{2,3 \#}$, Lucia Trevisan ${ }^{4}$, Raffaele Ferri $^{3}$, Rita Bella ${ }^{5}$ \\ ${ }^{1}$ Neurology Unit, Sant'Andrea Civic Hospital. La Spezia, Italy; ${ }^{2}$ Department of Surgery and Medical-Surgical Specialties, University of Catania, \\ Italy; ${ }^{3}$ Oasi Research Institute-IRCCS, Troina, Italy; ${ }^{4}$ Medical Genetic Unit, IRCCS Ospedale Policlinico San Martino, Genoa, Italy; ${ }^{5}$ Department \\ of Medical and Surgical Sciences and Advanced Technologies, University of Catania, Italy
}

\#These authors contributed equally to this work.

Correspondence to: Dr. Giuseppe Lanza, MD, PhD. Department of Surgery and Medical-Surgical Specialties, University of Catania, Via Santa Sofia, 78 - 95123, Catania, Italy. Email: glanza@oasi.en.it.

Submitted Aug 18, 2020. Accepted for publication Oct 27, 2020.

doi: 10.21037/qims-20-982

View this article at: http://dx.doi.org/10.21037/qims-20-982

\section{Introduction}

Parenchymal transcranial Doppler sonography (pTCS) has become a reliable and sensitive diagnostic technique, enabling a two-dimensional visualization of the main brain structures through the temporal bone (1). The growing application of this exam helps the diagnosis and differential diagnosis of a wide range of neurological diseases, especially movement disorders. pTCS is easily available and applicable, non-invasive, painless, cost effective, and quick to perform at the bedside, even in scarcely collaborating patients or in those with involuntary movements, because head motion can be well compensated by the operator, at least to some extent (2). These features make pTCS an useful additional tool for movement disorders, particularly in the case of challenging diagnoses or doubtful clinical presentations.

Subtle changes of specific deep brain structures, such as abnormal trace metal accumulation and circumscribed degenerative processes, can also be detected with pTCS (3). Moreover, the recently developed TCS devices offer the possibility to display some structures (e.g., basal ganglia, mesencephalic nuclei, width of the third ventricle, and the frontal horns of lateral ventricles) with a very high lateral and axial resolution (4).

Namely, substantia nigra (SN) hyperechogenicity has been proved to be a characteristic finding for idiopathic Parkinson's disease (PD), occurring in more than $90 \%$ of patients (5). Moreover, for early diagnosis of PD and differential diagnosis between PD and atypical parkinsonian syndromes, both the sensitivity and positive predictive value of SN hyperechogenicity were higher than $90 \%$, and both the specificity and negative predictive value were higher than $80 \%$ (5). TCS imaging of the $\mathrm{SN}$ also allows to differentiate between PD and essential tremor, with a high sensitivity (>90\%) and specificity (>89\%) (6). The diagnostic specificity further increased when combining pTCS findings of SN, lenticular nucleus (LN), and third ventricle width. In particular, despite some limitations, LN hyperechogenicity and third ventricle enlargement have been observed in some atypical parkinsonisms, such progressive supranuclear palsy, multiple system atrophy, and corticobasal degeneration (CBD) (7).

Despite these advantages, however, to date pTCS is still considered as an elective exam to perform in selected in-

^ ORCID: 0000-0002-5659-662X. 


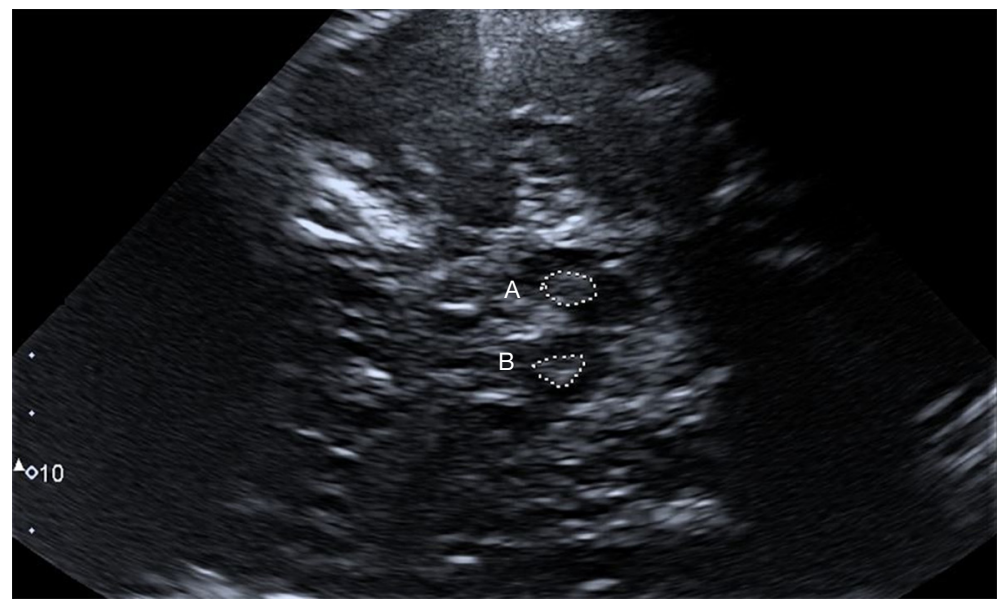

Figure 1 Marked bilateral hyperechogenicity of the substantia nigra. (A) Right side area, (B) left side area.

patients only, especially in those with movement disorders. Although movement disorders are commonly viewed as chronic diseases that are followed and treated in ambulatory care setting, a growing number of these patients present with acute or subacute disabling symptoms, as well as with complications of their underlying condition (8). Moreover, an acute movement disorder presenting at the Emergency Department (ED) points to a number of systemic, neurologic, drug-induced, or sometimes functional processes. A delayed diagnosis leads to a significant distress for the patients, with consequences on their management and quality of life (9).

Here, we report a patient with an unusual gait disorder in whom pTCS performed at the ED rapidly provided diagnostic findings towards a neurodegenerative movement disorder.

\section{Case report}

A 60-year-old man presented at the ED complaining of progressive walking difficulty for the last three-weeks, without falls. Family history was negative for neurological or psychiatric diseases. He worked as an electrician and lived alone. Because of a depressive disorder arisen one year earlier, he was under stable treatment with oral escitalopram $10 \mathrm{mg}$ and amisulpride $100 \mathrm{mg}$ daily. He was not taking any other medication, including the weeks before his presentation at the hospital.

As shown in the patient's recording, neurological examination showed an unusual gait characterized by ataxia, rigidity, and limping, with some instability but without falls (Video 1); he also had mild orofacial and hands dyskinesia, some of which were also present during walking, whereas the rest of the clinical examination was entirely normal. Notably, no motor impersistence, saccades/supranuclear gaze abnormality, or other signs of parkinsonism were present. Urgent laboratory tests, electrocardiogram, and computed tomography scan were normal. Based on the clinical picture of an undefined movement disorder in a patient taking a neuroleptic drug, a pTCS was performed at the ED.

TCS was carried out by using a phased array ultrasound system (Toshiba Aplio 300) equipped with a $2.5 \mathrm{MHz}$ transducer. The examination protocol was based on previous published recommendations for TCS (3). Using the transtemporal bone window approach, a B-mode image was obtained on both sides. First, the butterfly-shaped midbrain was visualized in axial untilted sections. Then, the scanning plane was shifted cranially to visualize diencephalic structures. As recommended (3), the $\mathrm{SN}$ was examined homolaterally to the insonation, whereas $\mathrm{LN}$ and caudate nucleus $(\mathrm{CN})$ contralaterally. Echogenicity and echotexture of $\mathrm{SN}$, thalami, $\mathrm{CN}$, and $\mathrm{LN}$ were analyzed bilaterally, and graded as hyperechogenic if were more intense than the surrounding white matter $(10,11)$. For each side, SN was evaluated quantitatively, whereas $\mathrm{LN}$ and $\mathrm{CN}$ were assessed semiquantitatively.

The pTCS investigation of this patient showed marked hyperechogenicity of the SN (Figure 1): A (right side) $=0.41 \mathrm{~cm}^{2} ; \mathrm{B}$ (left side) $=0.33 \mathrm{~cm}^{2}$ [cut-off values: area for mild hyperechogenicity $\geq 0.16 \mathrm{~cm}^{2}$; area for marked hyperechogenicity $\geq 0.22 \mathrm{~cm}^{2}$ (12)] and the $\mathrm{CN}$, bilaterally (Figure 2). This pattern, although not disease-specific, has been described in patients with Huntington's disease (HD). 


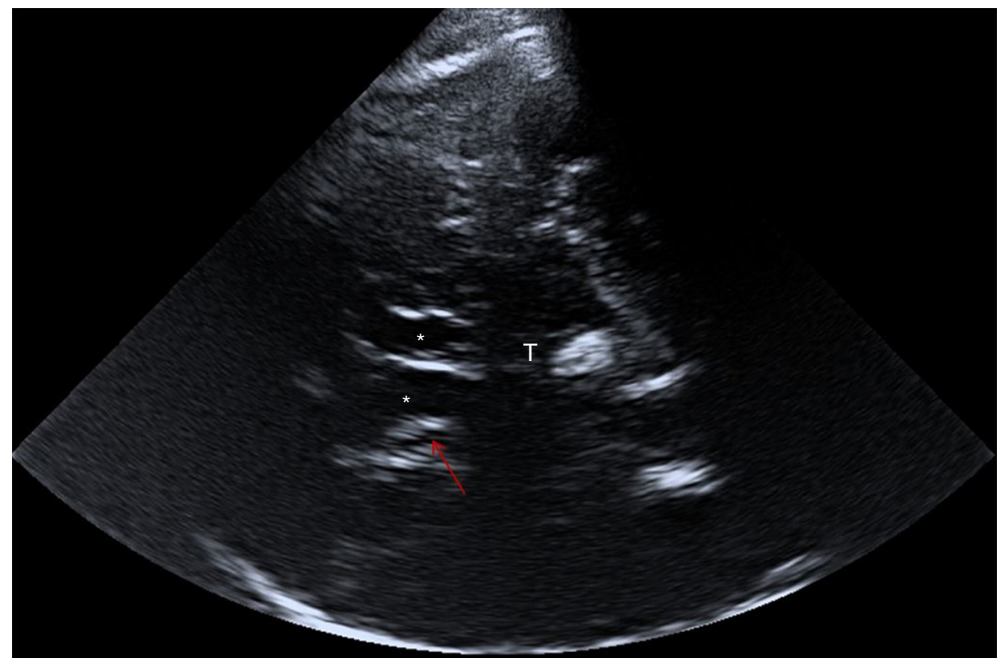

Figure 2 Normal size of the third ventricle (marked with "T") and lateral ventricles (frontal horns marked with asterisk); hyperechogenicity of the left caudate nucleus (arrow).

The third ventricle and the frontal horns of the lateral ventricles were normal [third ventricle size: $6 \mathrm{~mm}$, cut-off values for people $\geq 60$ years: $<10 \mathrm{~mm}$; frontal horns size: $15 \mathrm{~mm}$, cut-off values for people $\geq 60$ years: $<20 \mathrm{~mm}$ (3)].

Despite the lack of a family history, the patient age, and the referred recent onset of symptoms, HD was considered among the possible causes underlying this clinical presentation and, therefore, the patient was admitted to the Neurology Department. Laboratory tests (including serum copper and ceruloplasmin level, anti-streptolysin O, blood acanthocytes research), electroencephalogram, and 1.5-T brain magnetic resonance imaging (MRI) were normal. In particular, no striatal hyperintensity on T2-weighted MRI was noted. Molecular genetic test confirmed the diagnosis of HD, with one allele of the huntingtin (HTT) gene in chromosome $4 p 16.3$ showing an abnormal number of $40 \pm 1$ cytosine-adenine-guanine (CAG) repeats, whereas the number of CAG repeats in the other allele was within the normal limits $(24 \pm 1)$. During hospitalization, amisulpride was rapidly discontinued, but clinical manifestations did not improve and rather gradually worsened over time, as confirmed at the three-month clinical follow-up (Unified Huntington's Disease Rating Scale-motor assessmentat discharge: 13; score at follow-up: 15). In particular, the gait was more impaired, making the assistance necessary to avoid falling, whereas the rigidity was slightly decreased (especially at lower limbs).

The patient gave written informed consent for the publication of his personal and medical information, prior to the inclusion in the study, conformed to the provisions of the Declaration of Helsinki (as revised in 2013).

\section{Discussion}

To date, this is the first report of a HD sonographically detected at the ED. This supports the view that quantitative pTCS might be easily implemented in the diagnostic algorithms and differential diagnosis of movement disorders, even in an emergency setting.

$\mathrm{HD}$ is an autosomal dominant disorder caused by an unstable expansion of the trinucleotide CAG on chromosome $4 \mathrm{p} 16.3$, leading to a progressive degeneration of the basal ganglia, particularly of the neostriatum. The diagnosis focuses on typical involuntary movements, although the clinical presentation also includes cognitive decline and psychiatric symptoms (i.e., depression, irritability, anxiety, psychosis, and compulsive behavior). Psychiatric disturbances often precede the onset of motor symptoms (13), as in the present case, thus challenging an early diagnosis and treatment. Functional neuroimaging methods, such as single photon emission computed tomography and positron emission tomography, are able to detect functional changes, especially in the striatum, but they are expensive and not widely available (14). Notably, the MRI of our patient did not show any abnormalities of the SN nor hyperintensity of $\mathrm{CN}$, thus highlighting the diagnostic utility of quantitative pTCS in this case.

Although the frequency of late-onset HD ( $>59$ years) 
is assumed to be low and the clinical course milder, the previous literature is scarce and often inconclusive. A very recent study on a large cohort (15) founds that late-onset patients present more frequently with gait and balance problems as first symptoms, and the disease progression is not milder compared to common-onset HD patients, apart from motor progression. Moreover, in this patient subgroup, the family history is likely to be negative, which might make diagnosing HD more difficult, although balance and gait problems are helpful clinical clues (15). Taken together, these findings are in line with the patient described here; in this context, it is likely that gait and motor disorder had been present for more than three weeks, although neither the patient nor his family members were able to confirm that. It can be hypothesized that the assumption of amisulpride, an antidopaminergic drug, may have attenuated choreic movements, as recently reported (16).

The pTCS finding of SN hyperechogenicity indicates a functional impairment of the nigro-striatal dopaminergic system, and is assumed to be based on an increased amount of iron bound to proteins other than ferritin (3). This is also in agreement with molecular studies indicating the essential role of the huntingtin in cellular iron homeostasis. Although SN hyperechogenicity is characteristic for idiopathic $\mathrm{PD}$, it has been reported also in other conditions, such as atypical parkinsonisms, spinocerebellar ataxia, and HD $(10,17,18)$. In particular, Lambeck and colleagues (19) observed hyperechogenicity of the SN in 14 out of 15 patients with $\mathrm{HD}$, and, among them, seven showed bilateral hyperechogenicity.

We also can confirm the pathologic signal of the $\mathrm{CN}$ in $\mathrm{HD}$, a finding that supports the differentiation from other movement disorders (20). In previous studies $(10,11)$, an association between $\mathrm{CN}$ echogenicity and signal intensity in T2-weighted MRI in HD was reported. In particular, while SN hyperechogenicity was found to be significantly higher in the clinically severely affected patients, $\mathrm{CN}$ hyperechogenicity correlated with an increased signal intensity in T2-weighted MRI, but not with the disease severity (10). Based on these findings, it can be inferred that basal ganglia hyperechogenicity in HD may be correlated with changes of the iron-protein binding, which might cause echogenic alterations similar to the SN hyperechogenicity observed in PD patients $(3,18)$. Additionally, post mortem studies showed that not only iron, but also copper levels are increased in basal ganglia of HD subjects (21). However, the exact morphological and pathogenetic interpretation is still speculative and further neuropathological and multimodal imaging studies are needed.
Indeed, although the role of metals is assumed, the exact origin of hyperechogenicity has not been definitively confirmed, and the occurrence of structural changes can be conditioned by the presence of gliosis.

Regarding the LN hyperechogenicity, few earlier studies only are available, showing that this nucleus was less often affected or even spared in typical HD (10). LN seems to be more hyperechogenic in the hypokinetic-rigid form of HD (19), as well as in patients with dystonia and Wilson's disease (22), although none of these studies reported the specificity and sensitivity of the hyperechogenicity observed.

Finally, this patient was taking amisulpride, a benzamide blocking or antagonizing the presynaptic dopamine D2 receptor. As such, amisulpride is among the neuroactive compounds able to induce tardive dyskinesia, although the prevalence of amisulpride-induced movement disorder is substantially lower than that of the first-generation antipsychotic agents. Moreover, tardive dyskinesia commonly manifests with orofacial movements, tremor, dystonia, akathisia, and parkinsonism (23). Therefore, an iatrogenic cause, i.e., a drug-induced parkinsonism (DIP), needed to be taken into account on the basis on subacute onset, symmetric involvement, gait abnormality, and orofacial dyskinesia. However, the pTCS signs of a marked bilateral hyperechogenicity of both $\mathrm{SN}$ and $\mathrm{CN}$ did not support a DIP. Indeed, according to previous studies (24), although SN hyperechogenicity can be also observed in DIP, none of the DIP patients showed the CN hyperechogenicity. In the present case, we supposed that the improvement of rigidity and the worsening of gait might be correlated to the discontinuation of amisulpride and to the disease progression, respectively.

Although the application of pTCS in the ED setting is promising, the technique has some intrinsic limitations, such as the temporal acoustic bone window (insufficient in $5-10 \%$ of Caucasian individuals) (3), the degree of the operator expertise, and the quality of the ultrasound system. In this case, the exam was performed with a dedicated machine by a trained neurologist (MG) with an internationally-certified expertise in pTCS. Finally, it is worth reminding that, although SN and CN hyperechogenicity have been found in a higher proportion of HD patients than healthy controls (especially when parkinsonism was present), to date pTCS cannot provide specific findings of HD. Indeed, bilateral hyperechogenicity of the $\mathrm{SN}$ is also observed in $\mathrm{CBD}$ and in Lewy body Dementia, whereas $\mathrm{CN}$ hyperechogenicity has been linked to psychosis in PD $(25,26)$, as well as to some cases of multiple sclerosis (27), migraine (28), obsessive- 
compulsive disorder (29), and, more rarely, primary dystonia $(30,31)$. However, although these differential diagnoses cannot be excluded based on the pTCS alone, our patient did not have any clinical or radiological sign or history of the above-mentioned conditions. Overall, the use of pTCS in the differential diagnostics of hyperkinetic syndromes needs caution as the frequency of basal ganglia abnormalities detected by this technique is far from $100 \%$, both in HD and in other movement disorders (including those of autoimmune origin). Therefore, to date, pTCS cannot be viewed as a key tool in the diagnostics of HD, although it may support the diagnostic process and help the differential diagnosis of selected cases also in an Emergency setting.

\section{Conclusions}

In patients with clinically undefined movement disorders, pTCS can feasibly extend the neurological examination by providing diagnostic clues more rapidly and less expensively than neuroimaging. Cautious interpretation of the findings are mandatory and further systematic studies are needed to implement quantitative pTCS in clinical practice and to confirm the diagnostic utility of this technique in patients with movement disorders presenting at the ED.

\section{Acknowledgments}

We would like to thank the Italian Society of Neurosonology and Cerebral Hemodynamic (SINSEC) for education and training.

Funding: None.

\section{Footnote}

Conflicts of Interest: All authors have completed the ICMJE uniform disclosure form (available at http://dx.doi. org/10.21037/qims-20-982). The authors have no conflicts of interest to declare.

Ethical Statement: All procedures performed in studies involving human participants were in accordance with the ethical standards of the institutional research committee and with the Helsinki Declaration (as revised in 2013). Written informed consent was obtained from the patient for publication of this study and any accompanying images. A copy of the written consent is available for review by the Editor-in-Chief of this journal.
Open Access Statement: This is an Open Access article distributed in accordance with the Creative Commons Attribution-NonCommercial-NoDerivs 4.0 International License (CC BY-NC-ND 4.0), which permits the noncommercial replication and distribution of the article with the strict proviso that no changes or edits are made and the original work is properly cited (including links to both the formal publication through the relevant DOI and the license). See: https://creativecommons.org/licenses/by-nc-nd/4.0/.

\section{References}

1. Seidel G, Kaps M, Gerriets T, Hutzelmann A. Evaluation of the ventricular system in adults by transcranial duplex sonography. J Neuroimaging 1995;5:105-8.

2. Berg D, Godau J, Walter U. Transcranial sonography in movement disorders. Lancet Neurol 2008;7:1044-55.

3. Walter U, Behnke S, Eyding J, Niehaus L, Postert T, Seidel G, Berg D. Transcranial brain parenchyma sonography in movement disorders: state of the art. Ultrasound Med Biol 2007;33:15-25.

4. Walter U, Kanowski M, Kaufmann J, Grossmann A, Benecke R, Niehaus L. Contemporary ultrasound systems allow high-resolution transcranial imaging of small echogenic deep intracranial structures similarly as MRI: a phantom study. Neuroimage 2008;40:551-8.

5. Bor-Seng-Shu E, Paschoal FM, Almeida KJ, De Lima Oliveira M, Nogueira RC, Teixeira MJ, Walter U. Transcranial brain sonography for Parkinsonian syndromes. J Neurosurg Sci 2019;63:441-9.

6. Tao A, Chen G, Mao Z, Gao H, Deng Y, Xu R. Essential tremor vs idiopathic Parkinson disease: Utility of transcranial sonography. Medicine (Baltimore) 2020;99:e20028.

7. Alonso-Canovas A, TemblFerrairó JI, Martínez-Torres I, Lopez-Sendon Moreno JL, Parees-Moreno I, MonrealLaguillo E, Pérez-Torre P, Toledano Delgado R, García Ribas G, Sastre Bataller I, Masjuan J, Martinez-Castrillo JC, Walter U. Transcranial sonography in atypical parkinsonism: How reliable is it in real clinical practice? A multicentre comprehensive study. Parkinsonism Relat Disord 2019;68:40-5.

8. Lanza G, Papotto M, Pennisi G, Bella R, Ferri R. Epileptic seizure as a precipitating factor of vascular progressive supranuclear palsy: a case report. J Stroke Cerebrovasc Dis 2014;23:e379-81.

9. Dallocchio C, Matinella A, Arbasino C, Arno' N, Glorioso M, Sciarretta M, Braga M, Tinazzi M. Movement 
disorders in emergency settings: a prospective study. Neurol Sci 2019;40:133-8.

10. Postert T, Lack B, Kuhn W, Jergas M, Andrich J, Braun B, Przuntek H, Sprengelmeyer R, Agelink M, Büttner T. Basal ganglia alterations and brain atrophy in Huntington's disease depicted by transcranial real time sonography. J Neurol Neurosurg Psychiatry 1999;67:457-62.

11. Krogias C, Eyding J, Postert T. Transcranial sonography in Huntington's disease. Int Rev Neurobiol 2010;90:237-57.

12. Vivo-Orti MN, Tembl JI, Sastre-Bataller I, Vazquez-Costa JF, Sahuquillo P, Burguera JA. Evaluation of the substantia nigra by means of transcranial ultrasound imaging. Rev Neurol 2013;56:268-74.

13. Duff K, Paulsen JS, Beglinger LJ, Langbehn DR, Stout JC; Predict-HD Investigators of the Huntington Study Group. Psychiatric symptoms in Huntington's disease before diagnosis: the predict-HD study. Biol Psychiatry 2007;62:1341-6.

14. Niccolini F, Politis M. Neuroimaging in Huntington's disease. World J Radiol 2014;6:301-12.

15. Oosterloo M, Bijlsma EK, van Kuijk SM, Minkels F, de Die-Smulders CE. Clinical and genetic characteristics of late-onset Huntington's disease. Parkinsonism Relat Disord 2019;61:101-5.

16. Harris KL, Kuan WL, Mason SL, Barker RA. Antidopaminergic treatment is associated with reduced chorea and irritability but impaired cognition in Huntington's disease (Enroll-HD). J Neurol Neurosurg Psychiatry 2020;91:622-30.

17. Postert T, Eyding J, Berg D, Przuntek H, Becker G, Finger M, Schöls L. Transcranial sonography in spinocerebellar ataxia type 3. J Neural Transm Suppl 2004;68:123-33.

18. Walter U, Hoeppner J, Prudente-Morrissey L, Horowski S, Herpertz SC, Benecke R. Parkinson's disease-like midbrain sonography abnormalities are frequent in depressive disorders. Brain 2007;130:1799-807.

19. Lambeck J, Niesen WD, Reinhard M, Weiller C, Dose M, Zucker B. Substantia nigra hyperechogenicity in hypokinetic Huntington's disease patients. J Neurol 2015;262:711-7.

20. Walter U, Školoudík D. Transcranial sonography (TCS) of brain parenchyma in movement disorders: quality standards, diagnostic applications and novel technologies. Ultraschall Med 2014;35:322-31.

21. Bartzokis G, Tishler TA. MRI evaluation of basal ganglia ferritin iron and neurotoxicity in Alzheimer's and Huntingon's disease. Cell Mol Biol (Noisy-le-grand)
2000;46:821-33.

22. Bartova P, Skoloudik D, Bar M, Ressner P, Hlustik P, Herzig R, Kanovsky P. Transcranial sonography in movement disorders. Biomed Pap Med Fac Univ Palacky Olomouc Czech Repub 2008;152:251-8.

23. Martino D, Karnik V, Osland S, Barnes TRE, Pringsheim TM. Movement Disorders Associated With Antipsychotic Medication in People With Schizophrenia: An Overview of Cochrane Reviews and Meta-Analysis. Can J Psychiatry 2018;63:706743718777392.

24. López-Sendón Moreno JL, Alonso-Cánovas A, BuisánCatevilla J, García Barragán N, Corral Corral I, de Felipe Mimbrera A, Matute Lozano MC, Masjuan Vallejo J, Martínez-Castrillo JC. Substantia Nigra Echogenicity Predicts Response to Drug Withdrawal in Suspected Drug-Induced Parkinsonism. Mov Disord Clin Pract 2015;3:268-74.

25. Yilmaz R, Berg D. Transcranial B-Mode Sonography in Movement Disorders. Int Rev Neurobiol 2018;143:179-212.

26. Walter U, Dressler D, Wolters A, Wittstock M, Benecke R. Transcranial brain sonography findings in clinical subgroups of idiopathic Parkinson's disease. Mov Disord 2007;22:48-54.

27. Walter U, Wagner S, Horowski S, Benecke R, Zettl UK. Transcranial brain sonography findings predict disease progression in multiple sclerosis. Neurology 2009;73:1010-7.

28. Blažina K, Mahović-Lakušić D, Relja M. Brainstem nuclei changes in migraine detected by transcranial sonography. Neurol Sci 2017;38:1509-12.

29. Mavrogiorgou P, Nalato F, Meves S, Luksnat S, Norra C, Gold R, Juckel G, Krogias C. Transcranial sonography in obsessive-compulsive disorder. J Psychiatr Res 2013;47:1642-8.

30. Naumann M, Becker G, Toyka KV, Supprian T, Reiners K. Lenticular nucleus lesion in idiopathic dystonia detected by transcranial sonography. Neurology 1996;47:1284-90.

31. Hagenah J, König IR, Kötter C, Seidel G, Klein C, Brüggemann N. Basal ganglia hyperechogenicity does not distinguish between patients with primary dystonia and healthy individuals. J Neurol 2011;258:590-5.

Cite this article as: Godani M, Lanza G, Trevisan L, Ferri R, Bella R. An unusual gait disorder at the Emergency Department: role of the quantitative assessment of parenchymal transcranial Doppler sonography. Quant Imaging Med Surg 2021;11(5):2195-2200. doi: 10.21037/qims-20-982 